\title{
Unravelling regulatory networks
}

DOI:

10.1038/nrmicro1453

URLs

\section{Escherichia coli}

http://www.ncbi.nlm.nih.gov/ entrez/query.fcgi?db=genome prjधcmd=Retrieve\&dopt $=$ Over viewElist_uids $=12319$
Deciphering transcriptional regulatory interactions among genes, and their evolution, represents a major challenge for microbiologists in the post-genomic era. Obtaining a comparative overview of the transcriptional control of gene expression across a diverse range of microorganisms will allow researchers to elucidate the general principles that have shaped the evolution of this fundamental regulatory mechanism. In a recent issue of the Journal of Molecular Biology, M. Madan Babu and colleagues describe the computational reconstruction and comparative analysis of transcriptional regulatory networks for 175 phylogenetically diverse microorganisms.

The combined total of all transcriptional interactions in an organism - the transcriptional regulatory network - has been defined for Escherichia coli and this organism was used as the reference point to predict the components and structure of regulatory networks from the set of test genomes. Three levels of organization were assessed in this study. First, at the level of individual genes, the authors were able to show that transcription factors evolve rapidly, relative to their target genes. They also observed that microorganisms have independently evolved their own repertoire of transcription factors, indicating that an important factor in the adaptation of an organism to a new environment is the emergence of a distinct set of these proteins. At the level of local network structure, Babu et al. observed that organisms with similar lifestyles have similar regulatory interactions and have incorporated orthologous genes within similar patterns of network interconnections. At the global level, analysis of the data set revealed that conservation of transcription factors is independent of the number of their target genes, and also depends on the lifestyle of the organism, rather than their phylogenetic relatedness. It was also shown that different proteins have emerged independently to function as dominant transcription control 'hubs' in their organisms, and these transcription factors can be lost if they are not subject to selective pressure.

As the authors point out, their overall analysis suggests that, for a given organism, natural selection has 'tinkered' with individual interactions within the transcriptional regulatory network to arrive at a particular setup that is optimal for that organism. In the short term, the methods developed in this study can be applied to any set of networks and genomes, and have an important role in generating predictions that can be assessed experimentally. In the longer term, these observations have interesting implications for both our understanding of virulence gene regulation in pathogenic bacteria and in the ongoing search for new therapeutic targets.

David O'Connell

ORIGINAL RESEARCH PAPERS Babu, M. M. Teichmann, S. A. \& Aravind, L. Evolutionary dynamics of prokaryotic transcriptional regulatory networks.J. Mol. Biol. 358, 614-633 (2006) WEBSITES

M. Madan Babu's homepage: http://www2.mrcImb.cam.ac.uk/SS/babu_mm Sarah Teichmann's homepage: http://www2 mrc-Imb.cam.ac.uk/SS/Teichmann

L. Aravind's homepage: http://www.ncbi.nlm. nih.gov/CBBresearch/Aravind

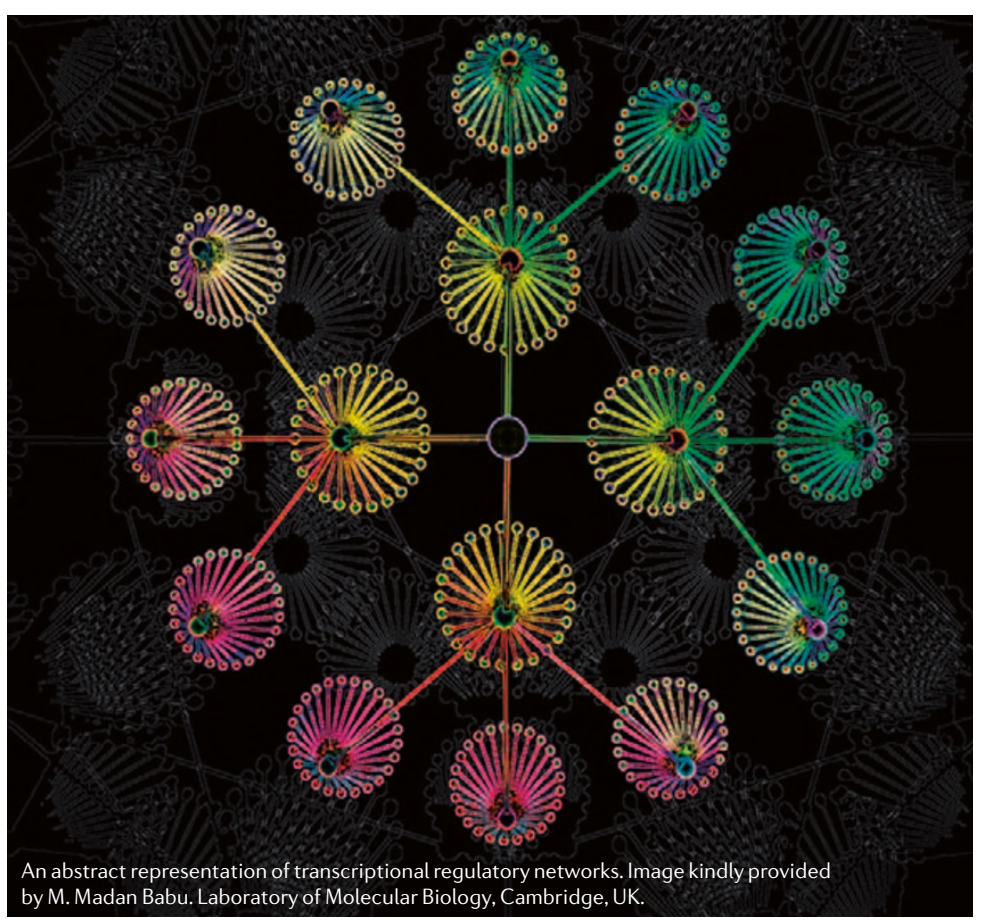

